\title{
Una experiencia sobre seguridad alimentaria para trabajar la argumentación en el aula de educación secundaria
}

\author{
Lucía Casas-Quiroga \\ Departamento de Didácticas Aplicadas, Área de Didáctica de las Ciencias Experimentales, Facultad de \\ Ciencias de la Educación, Universidade de Santiago de Compostela. Santiago de Compostela. España. \\ l.casas.quiroga@usc.es \\ ORCID: https:// orcid.org/0000-0003-0295-752X
}

Beatriz Crujeiras-Pérez

Departamento de Didácticas Aplicadas, Área de Didáctica de las Ciencias Experimentales, Facultad de Ciencias de la Educación, Universidade de Santiago de Compostela. Santiago de Compostela. España. beatriz:crujeiras@usc.es ORCID: bttps:/ / orcid.org/0000-0002-8333-6559

[Recibido: 22 Septiembre 2018. Revisado: 15 Noviembre 2018. Aceptado: 12 Diciembre 2018]

Resumen: En este trabajo se describe una experiencia en el aula encaminada a promover la participación del alumnado de secundaria en la práctica científica de argumentación, utilizando como contexto la seguridad alimentaria. Se presenta una secuencia de cuatro actividades para poner en práctica en los cursos $4^{\circ}$ de $\mathrm{ESO}$ y $1^{\circ}$ de Bachillerato en las asignaturas optativas de Ciencias Aplicadas para la Actividad Profesional (CAAP) y Cultura Científica (CC), que pretenden servir como recurso al profesorado de ciencias interesado en introducir el enfoque de aprendizaje a través de las prácticas científicas. La utilidad de la experiencia radica en la construcción del conocimiento por parte del alumnado a través de la reflexión y la justificación de cuestiones socio-científicas relacionadas con la seguridad alimentaria.

Palabras clave: Seguridad alimentaria; Educación secundaria; Prácticas científicas; Argumentación.

An experience on food safety to engage in argumentation in the high school

Abstract: This research describes a classroom experience aimed at engaging high school students in the scientific practice of argumentation, using food safety as framework. A sequence of four activities is presented, in order to be used in $10^{\text {th }}$ and $11^{\text {th }}$ grade in the optional subjects of Applied Sciences for Professional Activity and Scientific Culture. This pretends to be a resource for science teachers who are interested in the introduction of learning approaches through scientific practices. This experience proves its usefulness in that students are able to build knowledge through reflection and justification of socio-scientific issues related to food safety.

Keywords: Food safety; High school, Scientific practices; Argumentation.

Para citar este artículo: Casas-Quiroga, L., Crujeiras-Pérez, B., (2019) Una experiencia sobre seguridad alimentaria para trabajar la argumentación en el aula de educación secundaria. Revista Eureka sobre Enseñanza y Divulgación de las Ciencias 16(2), 2201. doi: 10.25267/Rev_Eureka_ensen_divulg_cienc.2019.v16.i2.2201

\section{Introducción}

El enfoque de aprendizaje a través de las prácticas científicas implica la participación del alumnado en el trabajo de construir el conocimiento en ciencias y de comprender porqué se elabora, prueba, evalúa y refina dicho conocimiento de la forma en la que se hace (Reiser, Berland y Kenyon, 2012). Esto motiva al alumnado a utilizar su conocimiento y a reflexionar sobre su propia comprensión acerca de las ideas científicas (NASEM, 2015).

En esta propuesta se fomenta dicha participación a través de la práctica de argumentación, entendida como el proceso de evaluación del conocimiento (teorías, hipótesis, explicaciones) 
en base a las pruebas disponibles (Jiménez Aleixandre, 2010). Mediante esta práctica el alumnado construye el conocimiento a través de la justificación, evaluación y cuestionamiento de cuestiones científicas y sociocientíficas (Jin et al., 2015). En este trabajo la argumentación se pone en práctica en el contexto de la seguridad alimentaria, en particular en la aplicación del marco de análisis para la intervención en situaciones de emergencia relativas a la inocuidad de los alimentos (FAO/OMS, 2011). Éstas incluyen riesgos biológicos, químicos y físicos asociados al consumo de alimentos. Este marco de análisis proporciona una forma sistemática de estimar los riesgos para identificar y poner en práctica medidas adecuadas para controlar los riesgos y comunicar la información sobre los mismos y sobre las medidas de control aplicadas. Aunque la seguridad alimentaria no se incluye como un tema específico a abordar en la educación secundaria, se considera un tema transversal de gran relevancia, ya que se presupone que mejorar las competencias en seguridad alimentaria de los ciudadanos permitiría disminuir la frecuencia de intoxicaciones alimentarias. Además, aunque estas cuestiones se abordan de forma directa en la educación superior, no se han encontrado trabajos que se centren en la aplicación explícita del marco de seguridad en ningún nivel educativo, sino que se centran en cuestiones biotecnológicas (Henderson et al., 1991), envasado (Jideani y Jideani, 2012) y en las propiedades organolépticas de los alimentos (Giacalone, 2016). Es por ello que consideramos de gran relevancia trabajar esta cuestión y las asignaturas optativas de CAAP y CC ofrecen una oportunidad idónea para ello.

\section{Descripción de la propuesta}

La experiencia consiste en la realización de una secuencia de cuatro actividades a lo largo de dos sesiones de 50 minutos, dirigidas a interpretar, analizar y evaluar los protocolos de seguridad que pueden llegar a activarse cuando tiene lugar una intoxicación alimentaria. Para ello, se escoge el marco de análisis de la OMS (Organización Mundial de la Salud) para brotes infecciosos, el cual detalla unas pautas de actuación a las que se tratará de dar sentido con la participación activa del alumnado en la resolución de las actividades diseñadas.

El estudio del marco de análisis de la OMS proporciona un contexto idóneo para introducir en el aula la importancia de una alimentación saludable, pero también segura. Errores en la manipulación de alimentos pueden generar la aparición de microorganismos y sustancias tóxicas responsables de las intoxicaciones alimentarias. Éstas pueden tener un mayor alcance en un mundo globalizado en el que se exportan e importan alimentos continuamente.

Teniendo en cuenta estos aspectos se diseña una secuencia de actividades de aula, la cual se describe en la figura 1.

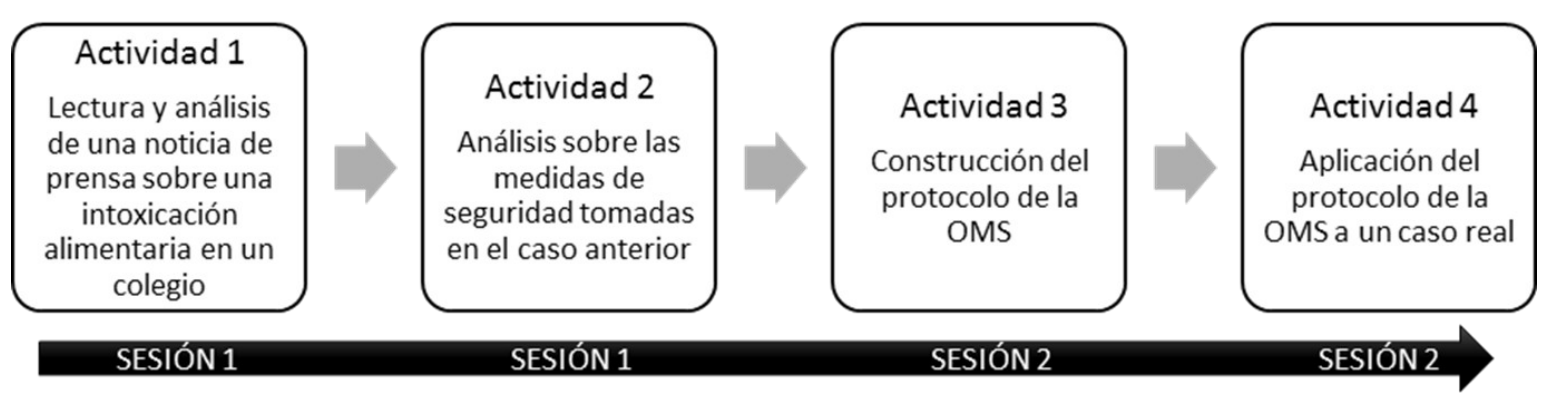

Figura 1. Secuencia de actividades diseñadas y distribución de las sesiones

Las actividades 1 y 2 se realizan de manera individual, mientras que para la 3 y 4 se forman los pequeños grupos. Esto nos permite diferenciar si el desempeño del alumnado cambia en 
ambas situaciones. En la primera sesión se realizan las dos primeras actividades y en la segunda las dos siguientes.

\section{Actividad 1}

Durante la actividad 1, cada estudiante lleva a cabo una lectura individual de una noticia de prensa cuyos hechos se sitúan en un colegio de Guadalajara en el que muchos estudiantes, usuarios del comedor escolar, presentan síntomas de vómitos y diarrea. Dicho contexto resulta próximo a los estudiantes, ya que se trata de un centro escolar. La noticia menciona que se barajan las alternativas de intoxicación alimentaria o virus. El objetivo de la actividad es estudiar el tipo de argumentos que utilizan los estudiantes y si incorporan los datos de la noticia para elaborar una justificación. Tras la lectura, los estudiantes deben contestar a una serie de preguntas que se recogen en la tabla 1, junto a las respuestas de referencia. Dichas cuestiones están relacionadas con la causa primera de los síntomas de los estudiantes del centro de Guadalajara y con las pruebas necesarias para demostrarlo. En la tabla 1 se resumen las respuestas de referencia para cada pregunta.

Tabla 1. Respuestas de referencia relativas a la Actividad 1

\begin{tabular}{|l|l|}
\hline \multicolumn{1}{|c|}{ Pregunta } & \multicolumn{1}{|c|}{ Respuesta de referencia } \\
\hline $\begin{array}{l}\text { 1. ¿Crees que se trata de una intoxicación } \\
\text { alimentaria o no? Explica por qué. }\end{array}$ & $\begin{array}{l}\text { Sí, ya que la noticia menciona que todos los } \\
\text { afectados comieron en el comedor escolar, por lo } \\
\text { que es lógico suponer que comieron algún } \\
\text { alimento en mal estado }\end{array}$ \\
\hline $\begin{array}{l}\text { 2. ¿Qué datos/pruebas necesitarías para poder } \\
\text { llegar a una conclusión? }\end{array}$ & $\begin{array}{l}\text { Para saber si es una intoxicación alimentaria, y no } \\
\text { un virus que proviene de otra fuente, es necesario } \\
\text { analizar las muestras recogidas en el comedor, } \\
\text { para ver si los alimentos tienen algún } \\
\text { microorganismo o toxina que también se } \\
\text { encuentra en las pruebas médicas hechas a los } \\
\text { afectados. También habría que comprobar si hubo } \\
\text { algún error en la manipulación de los alimentos. } \\
\text { Sería interesante comprobar si algún otro colegio } \\
\text { vivió una situación similar, ya que la noticia } \\
\text { menciona que el servicio de catering abastece a } \\
\text { muchos colegios de la zona }\end{array}$ \\
\hline
\end{tabular}

\section{Actividad 2}

La actividad 2 también está relacionada con la noticia de prensa. El procedimiento es análogo al anterior, y los estudiantes deben contestar a otras cuestiones relacionadas con las medidas de seguridad tomadas en el centro y su adecuación. La última cuestión se utiliza para introducir el marco de análisis de la OMS que utilizaremos en las actividades siguientes. El objetivo de la actividad es estudiar el tipo de justificaciones empleadas por los estudiantes y las decisiones que toman para solventar una alerta. Las preguntas y las respuestas de referencia se recogen en la tabla 2. 
Tabla 2. Respuestas de referencia relativas a la Actividad 2

\begin{tabular}{|c|c|}
\hline Pregunta & Respuesta de referencia \\
\hline $\begin{array}{l}\text { 1. ¿Qué medidas de seguridad se adoptaron en el } \\
\text { caso anterior? }\end{array}$ & $\begin{array}{l}\text { Recogida de muestras por parte de inspección } \\
\text { sanitaria } \\
\text { Inspección de la cocina y de las dependencias del } \\
\text { comedor } \\
\text { Aviso al alumnado de no ir al colegio si presentan } \\
\text { alguna sintomatología, y también acudir al médico } \\
\text { si es necesario }\end{array}$ \\
\hline 2. ¿Consideras que son adecuadas o no? & $\begin{array}{l}\text { La noticia no menciona si el servicio de comedor } \\
\text { está cerrado desde el incidente. Eso sería } \\
\text { importante si hubo algún error en la manipulación } \\
\text { de los alimentos que se puede seguir produciendo. } \\
\text { El resto de las medidas parecen adecuadas, ya que } \\
\text { se está investigando la causa de los síntomas de los } \\
\text { estudiantes. }\end{array}$ \\
\hline $\begin{array}{l}\text { 3. ¿Si fueses el responsable del servicio de } \\
\text { comedor como actuarías ante una alerta } \\
\text { alimentaria como la descrita en la noticia? }\end{array}$ & $\begin{array}{l}\text { Cancelaría el servicio de comedor hasta que se } \\
\text { esclareciese la situación, por si el origen de los } \\
\text { síntomas se debe a algún error en la manipulación } \\
\text { de los alimentos o a alguna dependencia del } \\
\text { comedor sin las medidas de higiene adecuadas. }\end{array}$ \\
\hline $\begin{array}{l}\text { 4. La OMS establece que todos los países } \\
\text { miembros deben notificar los brotes infecciosos } \\
\text { que ocurran dentro de sus fronteras y puedan } \\
\text { suponer un riesgo para la comunidad } \\
\text { internacional. Como sistema para medir el riesgo } \\
\text { de esos brotes, cuenta con un formulario con } \\
\text { cuatro preguntas. Si dos de las respuestas son } \\
\text { afirmativas, ha de comunicarse el brote a la OMS. } \\
\text { ¿Teniendo en cuenta los interrogantes, sería } \\
\text { necesario notificar la alerta a la OMS? } \\
\text { • ¿Tiene el brote un impacto serio } \\
\text { sobre la salud pública? } \\
\text { ¿ ¿Surgió el brote de forma } \\
\text { imprevista? } \\
\text { ¿ ¿Hay algún riesgo de que se } \\
\text { propague por distintos países? } \\
\text { • ¿Puede implicar la imposición } \\
\text { de restricciones al transporte } \\
\text { internacional de personas y mercancías? }\end{array}$ & $\begin{array}{l}\text { La única pregunta que se puede responder de } \\
\text { manera afirmativa es la segunda, ya que nada } \\
\text { presagiaba el brote producido. Sin embargo, el } \\
\text { impacto sobre la salud pública no es serio, ya que la } \\
\text { noticia habla de una sintomatología con diarreas } \\
\text { leves y en ningún caso se requirió hospitalización. } \\
\text { Parece que no hay riesgo de que se propague el } \\
\text { brote, ya que todos los casos se sitúan en el mismo } \\
\text { centro escolar. En cuanto al transporte } \\
\text { internacional de personas y mercancías ocurre lo } \\
\text { mismo: el brote está localizado, por lo que no son } \\
\text { necesarias esas restricciones. } \\
\text { No es necesario dar el aviso a la OMS. }\end{array}$ \\
\hline
\end{tabular}

\section{Actividad 3}

En la actividad 3, a cada pequeño grupo de alumnos se le entrega un conjunto de etiquetas que se corresponden con los pasos del marco de análisis de la OMS para brotes infecciosos, así como de una breve descripción de cada uno de los pasos. El grupo debe llegar a un consenso para ordenar los pasos de manera lógica, en la forma que consideren que funcionaría de manera más eficiente. Las etiquetas deberán ser colocadas sobre una cartulina y relacionadas con flechas. Conviene relacionar esta actividad con la última pregunta de la sesión anterior y decir que ahora nos hallamos en una situación diferente en la que sí es necesario dar el aviso. Para dirigirnos a los estudiantes utilizamos la palabra protocolo en lugar de marco de análisis, ya que este término, empleado en la versión inglesa del documento de la OMS es más intuitivo. Lo definimos como un "conjunto de normas que nos dicen cómo actuar ante ciertas situaciones". Es recomendable compararlo con algo más familiar para el alumnado, como el protocolo de evacuación de incendios del centro educativo o el protocolo que se activa 
durante un caso de acoso escolar. Por último, hay que recordar a los alumnos que la estructura del protocolo puede ser de cualquier tipo (lineal, en red) y que las flechas no tienen por qué ir en una única dirección. El objetivo de dicha actividad en relación con la práctica científica de argumentación es estudiar la clase de relaciones que establecen los estudiantes entre los pasos de protocolo y su justificación. El esquema de referencia, con el que se compararán los realizados por los estudiantes aparece en la figura 2.

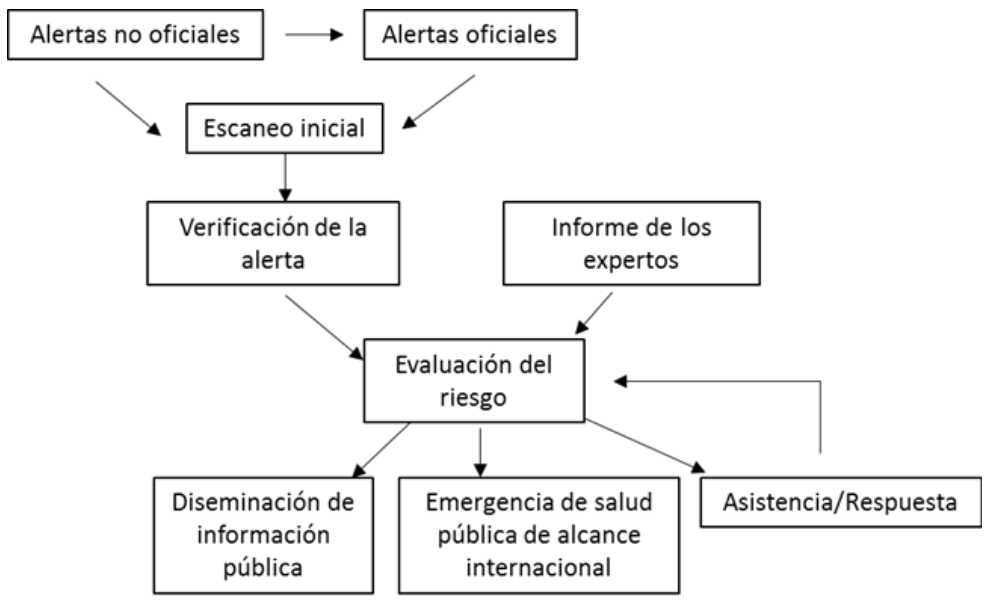

Figura 2. Marco de análisis de la OMS para brotes epidémicos

\section{Actividad 4}

Durante la actividad 4, se les presenta a los alumnos un caso real (el brote del síndrome urémico-hemolítico de Alemania en 2011) en el que el marco de análisis de la OMS no fue aplicado correctamente. Cada grupo debe reflexionar y argumentar en base a la información disponible cuáles fueron los pasos que se realizaron de manera errónea, y cuáles son las pruebas más relevantes que ayudaron a esclarecer el caso. Las preguntas y respuestas de referencia se recogen en la tabla 3.

Tabla 3. Respuestas de referencia relativas a la actividad 4

\begin{tabular}{|l|l|}
\hline \multicolumn{1}{|c|}{ Pregunta } & \multicolumn{1}{|c|}{ Respuesta de referencia } \\
\hline $\begin{array}{l}\text { 1. ¿Consideráis que la forma de proceder del } \\
\text { gobierno alemán es adecuada desde el punto de } \\
\text { vista científico? Justifica la respuesta }\end{array}$ & $\begin{array}{l}\text { No, ya que la senadora comparece en los medios } \\
\text { de comunicación señalando a los pepinos } \\
\text { españoles como causantes del brote sin ningún } \\
\text { análisis que lo confirme. También se hace la } \\
\text { recomendación de no consumir hortalizas después } \\
\text { de encontrar E.Coli, pero sin confirmar que esa } \\
\text { cepa de bacterias fue la que provocó el brote. Esto } \\
\text { afecta a los productores y distribuidores de esos } \\
\text { productos. }\end{array}$ \\
\hline $\begin{array}{l}\text { 2. ¿Qué pruebas de las indicadas consideráis más } \\
\text { relevantes para resolver el problema de seguridad } \\
\text { alimentaria? ¿Por qué? }\end{array}$ & $\begin{array}{l}\text { Los análisis realizados en el laboratorio en los que } \\
\text { se comparan las bacterias encontradas en los } \\
\text { alimentos (pepinos y soja) con las de las heces de } \\
\text { los intoxicados para determinar que cepa coincide. } \\
\text { Si los intoxicados consumieron ese alimento y la } \\
\text { cepa de bacterias coincide, tenemos el origen de la } \\
\text { intoxicación. }\end{array}$ \\
\hline
\end{tabular}




\begin{tabular}{|l|l|}
$\begin{array}{l}\text { 3. ¿ंAnalizando el protocolo seguido, identificáis } \\
\text { algún fallo? Indicad cuál o cuáles }\end{array}$ & $\begin{array}{l}\text { La senadora alemana compareciendo en los medios } \\
\text { señalando como originarios del brote a pepinos } \\
\text { españoles sin tener pruebas. } \\
\text { La recomendación por parte del gobierno alemán } \\
\text { de no consumir frutas yortalizas procedentes de } \\
\text { España sin tener la confirmación de que sean los } \\
\text { causantes del brote. }\end{array}$ \\
\hline
\end{tabular}

\section{Implementación de la propuesta}

La experiencia se realiza en $4^{\circ}$ de ESO, en la asignatura de Ciencias Aplicadas para la Actividad Profesional (CAAP) y en $1^{\circ}$ de BAC, en la asignatura de Cultura Científica (CC). En $4^{\circ}$ de ESO participa un grupo de ocho estudiantes que presentan dificultades asociadas con el aprendizaje de las ciencias. Para las actividades 3 y 4 se organizan en dos grupos de trabajo de cuatro integrantes. En $1^{\circ}$ de BAC participa un grupo de ocho estudiantes, cuatro de la rama de ciencias sociales y cuatro de la rama de ciencias. Se utiliza este mismo criterio para formar dos grupos de cuatro integrantes en las actividades 3 y 4 .

La secuencia de actividades fue introducida por el docente habitual de cada materia, acudiendo el alumnado a ellos para la resolución de dudas. El papel de la investigadora se limitó a la observación con participación moderada en el caso de necesitarse aclaraciones. Cabe señalar que los participantes no trabajaron previamente cuestiones argumentativas de forma explícita, sino que se abordaron de forma implícita con la realización de las tareas de la propia secuencia.

A continuación, se comentarán los aspectos más destacados del desempeño de los estudiantes durante la implementación de la experiencia, haciendo hincapié en las diferencias observadas entre los dos cursos participantes.

En primer lugar, en lo relativo a la actividad 1, la comprensión de la noticia de prensa es buena. Casi la totalidad de los estudiantes de ambos cursos argumenta que el hecho de que los estudiantes afectados fuesen usuarios del comedor escolar es un indicativo de que se ha producido una intoxicación alimentaria, y no el contagio mediante virus. En lo referente a los datos necesarios para llegar a una conclusión, la mayor parte de los estudiantes menciona únicamente la recogida de muestras, aunque dos alumnos de $1^{\circ}$ de $\mathrm{BAC}$ hacen uso de otros datos, como el análisis de las dependencias del comedor y las pruebas médicas.

Durante la actividad 2 se siguen unas pautas similares. En la cuestión referente a las medidas de seguridad tomadas casi todos los estudiantes de $1^{\circ}$ de BAC son capaces de mencionar todas ellas (recogida de muestras, análisis del comedor y de la cocina y aviso de acudir al médico), mientras que los alumnos de $4^{\circ}$ de ESO olvidan por completo el análisis del comedor y la cocina. En general, los estudiantes de ambos cursos están de acuerdo con la adecuación de las medidas a la situación descrita en la noticia, aunque, en caso de actuar ellos como responsables del servicio de comedor, varios proponen otras medidas como el cierre del servicio de comedor hasta esclarecer lo ocurrido para evitar más casos (mencionado por 4 alumnos de $1^{\circ}$ de BAC), la asunción de responsabilidades por parte del centro (mencionado por 3 alumnos de $4^{\circ}$ de ESO y 2 alumnos de $1^{\circ}$ de BAC) y mayores controles a los alimentos servidos para que la situación no se repita (mencionado por 1 alumno de $1^{\circ}$ de $\mathrm{BAC}$ ).

Sin embargo, en lo relativo al cuestionario de la OMS para dar aviso de brotes infecciosos, un mayor porcentaje de alumnos de $4^{\circ}$ de $\operatorname{ESO}\left(57^{\%}\right)$ frente a los de $1^{\circ}$ de $\mathrm{BAC}(14 \%)$ fueron capaces de argumentar correctamente que dicho aviso no era necesario respondiendo solamente a la segunda cuestión de manera afirmativa. En ambos cursos, los errores 
cometidos a la hora de responder el cuestionario provienen de una valoración incorrecta de la gravedad de la sintomatología de los afectados, ya que en este caso no se puede considerar que el impacto sobre la salud pública sea serio. El alumnado consideró que a pesar de que la sintomatología no parecía de gravedad, el hecho de que hubiese muchos estudiantes afectados (unos 80 según la noticia) afectaba a la salud pública, lo que revela cierta confusión con dicho término.

En la construcción del marco de análisis de la OMS durante la actividad 3, se obtuvieron resultados muy dispares entre los estudiantes de CC y CAAP, y también entre los grupos de un mismo curso.

En el caso del alumnado de $4^{\circ}$ de ESO, como se puede ver en la figura 3, existe confusión en el orden de los pasos del marco de análisis. El grupo B, por ejemplo, argumenta que tiene mayor importancia alertar a la población para que sepan cómo actuar (diseminación de información pública) antes de hacer las comprobaciones exhaustivas que permitirían valorar la gravedad de la situación (informe de los expertos).

Un error que cometen los dos grupos es proponer una estructura completamente lineal, en la que ningún paso genera diferentes vías de actuación.

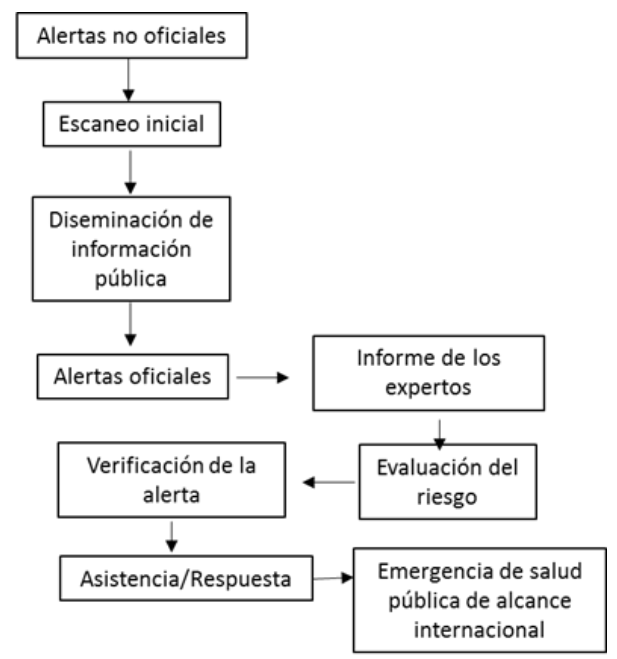

Figura 3. Protocolo elaborado por estudiantes de $4^{\circ}$ de ESO. Grupo B

Los marcos de análisis elaborados por los estudiantes de $1^{\circ}$ de $\mathrm{BAC}$ son más parecidos al marco de análisis de referencia, lo que se traduce en justificaciones como la necesidad de hacer todas las comprobaciones pertinentes sobre la enfermedad y su alcance antes de alarmar a la población o la necesidad de que los organismos no oficiales como los colegios puedan contactar con los oficiales antes de dar la alerta, pues los últimos están más preparados. Ambos marcos de análisis tienen una estructura más reticular y, aunque se observa cierta confusión entre algunos pasos, muchas de las relaciones establecidas son correctas. En el caso del grupo A (ciencias), los cuatro primeros pasos del marco de análisis están colocados de manera análoga al marco de análisis de referencia, como se puede observar en la figura 4. 


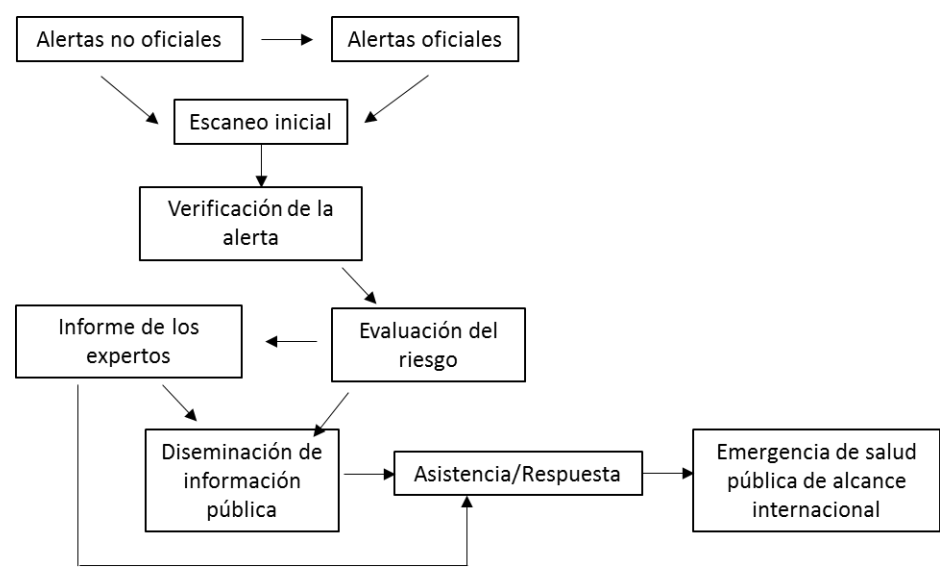

Figura 4. Protocolo elaborado por estudiantes de $1^{\circ}$ de BAC. Grupo A (ciencias)

La actividad 4 es en la que se observan mayores discrepancias entre los dos cursos que participan en la experiencia. Las respuestas aportadas por los estudiantes de CAAP denotan una comprensión pobre de la actividad, ya que ambos grupos ponen de manifiesto que el gobierno alemán actuó de forma correcta dando el aviso a la prensa. Esto puede relacionarse con el marco de análisis elaborado en la actividad anterior, en el que el grupo B situaba la diseminación de información pública como un paso inicial. Sin embargo, ambos grupos identifican fallos en el marco de análisis relacionados con el hecho de señalar culpables sin hacer los análisis pertinentes.

El alumnado de CC sí identifica una manera errónea de proceder del gobierno alemán, y argumentan que se afirmó la procedencia del brote sin investigarlo (grupo A, ciencias) o se dio por cierta una información no verificada (grupo B, ciencias sociales), lo que tuvo consecuencias para los productores.

En lo referente a las pruebas más relevantes para resolver el problema y en los fallos del protocolo, en ambos cursos destaca la parquedad de las respuestas, posiblemente asociada a la falta de tiempo para finalizar la actividad. Pese a eso, se identifica como prueba más importante los análisis de las heces de los afectados, aunque la justificación es muy escasa.

\section{Conclusiones e implicaciones educativas}

En este artículo se describe una experiencia en aula en la que se analizan los desempeños del alumnado relativos a la práctica de argumentación. Los resultados obtenidos reflejan desempeños dispares en ambos cursos, un hecho esperable por las diferentes características de los mismos. Las justificaciones elaboradas por los estudiantes de $1^{\circ}$ de $\mathrm{BAC}$ fueron elaboradas y más acordes a las respuestas de referencia, aunque en la primera sesión, en la que se realizaron las actividades 1 y 2 , el desempeño entre ambos cursos difiere en menor medida.

Esta secuencia de actividades ofrece la oportunidad de desarrollar la práctica científica de la argumentación, a la vez que se exploran conceptos relacionados con la seguridad alimentaria (intoxicación, OMS, protocolo...), una temática transversal que no se trata habitualmente en el aula, pese a su relevancia para la totalidad de la población.

Teniendo en cuenta que el diseño de la secuencia obedece a la necesidad de trabajar la argumentación en el aula, se observó en los estudiantes de $1^{\circ}$ de $\mathrm{BAC}$ una mayor disposición para adaptarse a un tipo de trabajo diferente, mientras que los estudiantes de $4^{\circ}$ de ESO tuvieron más dificultades, lo que sugiere que para dicho curso sería necesaria una adaptación 
de la temporalización de las actividades o de una mayor intervención del docente durante el todo el proceso.

\section{Agradecimientos}

Este estudio está financiado por el proyecto EDU2017-82915-R, del Ministerio de Ciencia, Innovación y Universidades y cofinanciado por el Fondo Europeo de Desarrollo Regional (AEI/FEDER, UE). Las autoras agradecen a los alumnos, alumnas y profesores que participaron en el estudio.

\section{Referencias Bibliográficas}

Giacalone, D. (2016). Enhancing Student Learning with Case-Based Teaching and Audience Response Systems in an Interdisciplinary Food Science Course. Higher Learning Research Communications, 6 (3).

Henderson, J., Knutton, S., Lally, V., Windale, M. (1991). Biotechnology and the food industry. Journal of Biological Education, 25, 95-102.

Jideani, V. A., Jideani, I. A. (2012). Alignment of Assessment Objectives with Instructional Objectives Using Revised Bloom's Taxonomy-The Case for Food Science and Technology Education. Journal of Food Science Education, 11, 34-42.

Jiménez Aleixandre, M.P. (2010). 10 Ideas Clave. Competencias en argumentación y uso de pruebas. Barcelona: Graó.

Jin, H., Mehl, C. E., Lan, D. H. (2015). Developing an Analytical Framework for Argumentation on Energy Consumption Issues. Journal of Research in Science Teaching, 52(8), 1132-1162.

National Academies of Sciences, Engineering, and Medicine (NASEM) (2015). Science Teachers Learning: Enhancing Opportunities, Creating Supportive Contexts. Committee on Strengthening Science Education through a Teacher Learning Continuum. Board on Science Education and Teacher Advisory Council, Division of Behavioral and Social Science and Education. Washington, DC: The National Academies Press.

Organización de las Naciones Unidas para la Agricultura y la Alimentación/Organización Mundial de la Salud (FAO/OMS). (2011). Guia FAO/OMS para la aplicación de principios y procedimientos de análisis de riesgos en situaciones de emergencia relativas a la inocuidad de los alimentos. Roma: FAO/OMS.

Reiser, B. J., Berland, L. K., Kenyon, L. (2012). Engaging Students in Scientific Practices of Explantion and Argumentation. Science and Children, 49(8), 8-13. 